\title{
Automated Verification of Shape, Size and Bag Properties
}

\author{
Wei-Ngan Chin ${ }^{1,2} \quad$ Cristina David $^{1} \quad$ Huu Hai Nguyen ${ }^{2}$ Shengchao Qin ${ }^{3}$ \\ ${ }^{1}$ Department of Computer Science, National University of Singapore \\ ${ }^{2}$ Computer Science Programme, Singapore-MIT Alliance \\ ${ }^{3}$ Department of Computer Science, Durham University \\ \{chinwn,davidcri,nguyenh2\}@comp.nus.edu.sg shengchao.qin@durham.ac.uk
}

\begin{abstract}
In recent years, separation logic has emerged as a contender for formal reasoning of heap-manipulating imperative programs. Recent works have focused on specialised provers that are mostly based on fixed sets of predicates. To improve expressivity, we have proposed a prover that can automatically handle user-defined predicates. These shape predicates allow programmers to describe a wide range of data structures with their associated size properties. In the current work, we shall enhance this prover by providing support for a new type of constraints, namely bag (multiset) constraints. With this extension, we can capture the reachable nodes (or values) inside a heap predicate as a bag constraint. Consequently, we are able to prove properties about the actual values stored inside a data structure.
\end{abstract}

\section{Introduction}

Separation logic supports reasoning about shared mutable data structures. Therefore, specification of memory operations with advanced pointer usage is enabled. While the foundations of separation logic have been laid in seminal papers by Reynolds [23] and Isthiaq and O'Hearn [11], new automated reasoning tools based on separation logic, such as $[2,9]$, are beginning to appear. Several recent works, such as $[1,8]$, have developed specialised solvers that work for a fixed set of predicates (e.g. 1seg). The level of expressivity for an automated verification system was raised by our previous work [20] where we support automated reasoning of user-defined predicates. The main goal of our current paper is to enhance the prover proposed in [20] by providing support for bag constraints. We make the following technical contributions towards this overall goal :

- We provide a shape predicate specification mechanism that can capture a wide range of data structures together with size and bag properties, such as various height-balanced trees, priority heap, sorted list, etc. Moreover, we provide a mechanism to soundly approximate each shape predicate by a heap-independent invariant which plays an important role in entailment checking (Secs 2 and 4.1).

- We improve the expressiveness of our automatic verification tool by allowing it to capture both the size properties and the bag of values from each shape predicate. The numeric properties capture sophisticated data structure invariants, such as orderedness (for sorted list/trees) and also balanced height properties (for AVL-trees). The bag constraints enable expressing reachability properties, such as the preservation of the elements inside a list after sorting.

- We design a new procedure to check entailment of separation heap constraints. This procedure uses unfold/fold reasoning to deal with shape definitions. While the unfold/fold mechanism is not new, we have identified sufficient conditions for soundness and termination of the procedure in the presence of recursive user-defined shape predicates. (Secs 3.1, 4 and 5)

- We have implemented a prototype verification system with the above features and have also proven both its soundness and termination (Secs 6 and 7).

\section{User-Definable Shape Predicates}

Separation logic $[23,11]$ extends Hoare logic to support reasoning about shared mutable data structures. It adds two more connectives to classical logic : separating conjunction $*$, and separating implication $\rightarrow$. $h_{1} * h_{2}$ asserts that two heaps described by $h_{1}$ and $h_{2}$ are domain-disjoint. $h_{1}-h_{2}$ asserts that if the current heap is extended with a disjoint heap described by $h_{1}$, then $h_{2}$ holds in the extended heap. In this paper we use only separating conjunction.

We propose an intuitive mechanism based on inductive predicates (or relations) to allow user specification of 
shapely data structures with size and reachability properties. Our shape specification is based on separation logic with support for disjunctive heap states. Furthermore, each shape predicate may have pointer, integer or bag parameters to capture relevant properties of data structures. We use the following data node declarations for the examples in the paper. They are recursive data declarations with different number of fields.

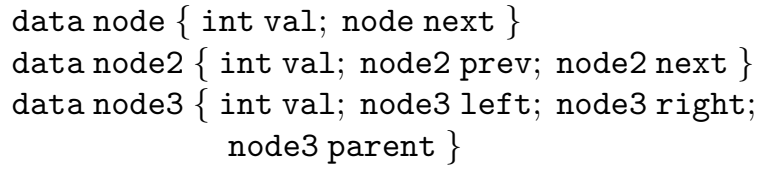

We use $p:: c\left\langle v^{*}\right\rangle$ to denote two things in our system. When $c$ is a data name, $\mathrm{p}: \mathrm{c}\left\langle\mathrm{v}^{*}\right\rangle$ stands for singleton heap $\mathrm{p} \longmapsto\left[(\mathrm{f}: \mathrm{v})^{*}\right]$ where $\mathrm{f}^{*}$ are fields of data declaration $\mathrm{c}$. When $c$ is a predicate name, $\mathrm{p}:: \mathrm{c}\left\langle\mathrm{v}^{*}\right\rangle$ stands for the formula $c\left(p, v^{*}\right)$. The reason we distinguish the first parameter from the rest is that each predicate has an implicit parameter self as the first one. Effectively, self is a "root" pointer to the specified data structure that guides data traversal and facilitates the definition of well-founded predicates (Sec 3.1). As an example, a singly linked list with length $\mathrm{n}$ is described by :

$$
\begin{aligned}
l l\langle\mathrm{n}\rangle \equiv(\operatorname{self} & =\mathrm{null} \wedge \mathrm{n}=0) \vee(\exists \mathrm{i}, \mathrm{m}, \mathrm{q} \cdot \operatorname{self}: \text { node }\langle\mathrm{i}, \mathrm{q}\rangle \\
& * \mathrm{q}:: l \mathrm{l}\langle\mathrm{m}\rangle \wedge \mathrm{n}=\mathrm{m}+1) \quad \text { inv } \mathrm{n} \geq 0
\end{aligned}
$$

Note that the parameter $n$ captures a derived value. The above definition asserts that an 11 list can be empty (the base case $\operatorname{self}=\mathrm{null}$ ) or consists of a head data node (specified by self::node $\langle i, q\rangle$ ) and a separate tail data structure which is also an 11 list ( $\mathrm{q}:: 11\langle\mathrm{~m}\rangle)$. The $*$ connector ensures that the head node and the tail reside in disjoint heaps. We also specify a default invariant $n \geq 0$ that holds for all 11 lists. Our predicate uses existential quantifiers for local values and pointers, such as i, m, q.

A more complex shape, doubly linked-list with length $n$, is described by :

$$
\begin{aligned}
\mathrm{dll} l \mathrm{p}, \mathrm{n}\rangle & \equiv(\operatorname{self}=\mathrm{null} \wedge \mathrm{n}=0) \vee(\operatorname{self}:: \operatorname{node} 2\langle-, \mathrm{p}, \mathrm{q}\rangle \\
& * \mathrm{q}:: \mathrm{dll}\langle\langle\operatorname{self}, \mathrm{n}-1\rangle) \quad \operatorname{inv} \mathrm{n} \geq 0
\end{aligned}
$$

The $\mathrm{dll}$ shape predicate has a parameter $\mathrm{p}$ that represents the prev field of the first node of the doubly linked-list. It captures a chain of nodes that are to be traversed via the next field starting from the current node self. The nodes accessible via the prev field of the self node are not part of the dll list. This example also highlights some shortcuts we may use to make shape specification shorter. We use underscore _ to denote an anonymous variable. Non-parameter variables in the RHS of the shape definition, such as q, are considered existentially quantified. Furthermore, terms may be directly written as arguments of shape predicate or data node.
User-definable shape predicates provide us with more flexibility than some recent automated reasoning systems $[1,3]$ that are designed to work with only a small set of fixed predicates. Furthermore, our shape predicates can describe not only the shape of data structures, but also their size and bag properties. This capability enables many applications, especially to support data structures with sophisticated invariants. For example, we may define a non-empty sorted list as below. The predicate also tracks the length, the minimum and maximum elements of the list.

$$
\begin{aligned}
& \text { sortl }\langle\mathrm{n}, \min , \max \rangle \equiv \\
& \quad(\operatorname{self}:: \operatorname{node}\langle\min , \operatorname{null}\rangle \wedge \min =\max \wedge \mathrm{n}=1) \\
& \vee(\operatorname{self}:: \operatorname{node}\langle\min , \mathrm{q}\rangle * \mathrm{q}:: \operatorname{sortl}\langle\mathrm{n}-1, \mathrm{k}, \max \rangle \wedge \min \leq \mathrm{k}) \\
& \quad \operatorname{inv} \min \leq \max \wedge \mathrm{n} \geq 1
\end{aligned}
$$

The constraint $\min \leq \mathrm{k}$ guarantees that sortedness property is adhered between any two adjacent nodes in the list. We may now specify (and then verify) the following insertion sort algorithm :

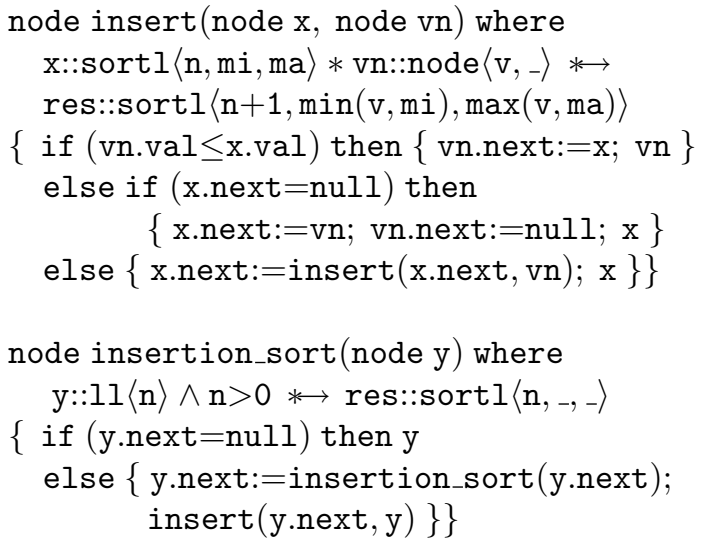

We use the notation $\Phi_{p r} * \Phi_{p o}$ to capture a precondition $\Phi_{p r}$ and a postcondition $\Phi_{p o}$ of a method. Note that we use an expression-oriented language where the last subexpression (e.g. $e_{2}$ from $e_{1} ; e_{2}$ ) denotes the result of an expression. A special identifier res is used in the postcondition to denote the result of a method. The postcondition of insertion_sort shows that the output list is sorted and has the same number of nodes as the input list.

\subsection{Bag of Values/Addresses}

The earlier specification of sorting captures neither the in-situ reuse of memory cells nor the fact that all the elements of the list are preserved by sorting. The reason is that the shape predicate captures only pointers and numbers but does not capture the set of reachable nodes in a heap predicate. A possible solution to this problem is to extend our specification mechanism to capture either a set or a bag of values. For generality and simplicity, we propose to use only the bag (or multi-set) notation that permits 
duplicates, though set notation could also be supported. In the rest of the paper, we will use the following bag operators: bag union $\sqcup$, bag intersection $\sqcap$, bag subsumption $\sqsubset$, and bag cardinality $|B|$. The shape specifications from the previous section are revised as follows:

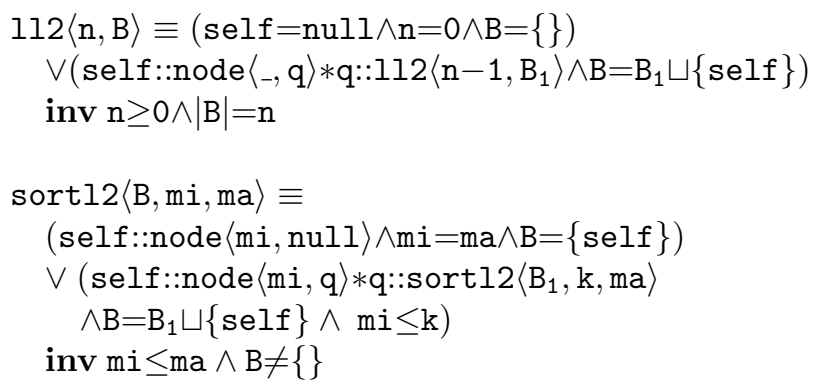

Each predicate of the form $112\langle\mathrm{n}, \mathrm{B}\rangle$ or sortl2〈B,mi,ma now captures a bag of addresses $B$ for all the data nodes of its data structure (or heap predicate). With this extension, we can provide a more comprehensive specification for in-situ sorting, as follows :

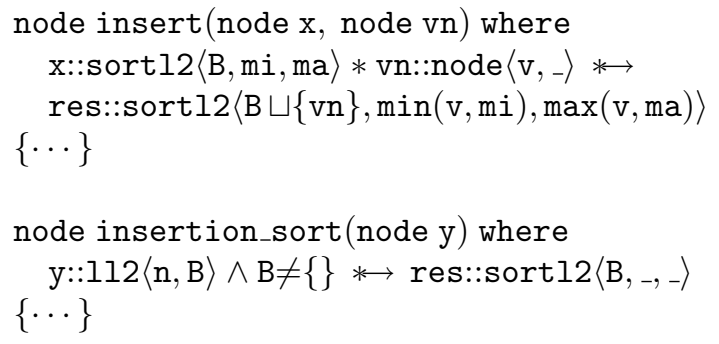

We stress that the bag mechanism used to capture the reachable nodes in a shape predicate is quite general. Instead of heap addresses, we may also revise our views to capture a bag of reachable values, as well as the length. For example:

$$
\begin{aligned}
& 113\langle\mathrm{n}, \mathrm{B}\rangle \equiv(\operatorname{self}=\mathrm{null} \wedge \mathrm{n}=0 \wedge \mathrm{B}=\{\}) \vee \\
& \quad\left(\text { self::node }\langle\mathrm{a}, \mathrm{q}\rangle * \mathrm{q}:: 113\left\langle\mathrm{n}-1, \mathrm{~B}_{1}\right\rangle \wedge \mathrm{B}=\mathrm{B}_{1} \sqcup\{\mathrm{a}\}\right) \\
& \text { inv } \mathrm{n} \geq 0 \wedge|\mathrm{B}|=\mathrm{n}
\end{aligned}
$$

Capturing a bag of values allows us to reason about the collection of values in a data structure, and permits relevant properties to be automatically verified. Both universal and existential properties over bags may be expressed and proven, when implemented with an appropriate constraint solver, as highlighted below :

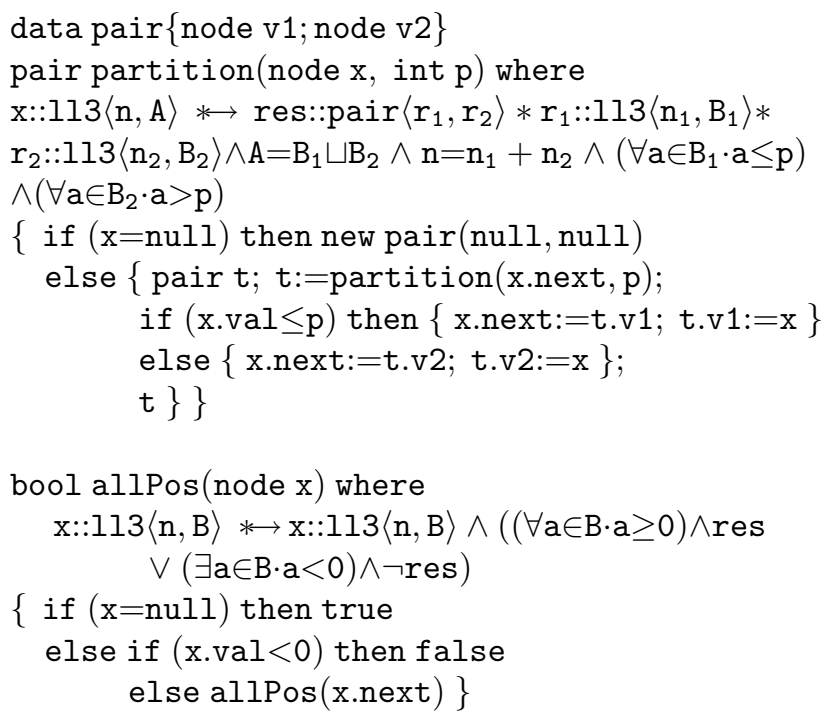

The first example returns a pair of lists that have been partitioned from a single input list according to an integer pivot. This partition function and its pre/post specification can be used to prove the total correctness of quicksort algorithm. The second example uses existentially and universally quantified formulae to determine if at least one negative number is present in an input list, or not.

\section{Automated Verification}

In this section, we first introduce a core object-based imperative language and then propose a set of forward verification rules to systematically check that preconditions are satisfied at call sites, and that the declared postcondition is successfully verified (assuming the precondition) for each method definition.

\subsection{Language}

We provide a simple imperative language in Figure 1. Our language is strongly typed and we assume programs and constraints are well-typed. The language supports data type declaration via datat, and shape predicate definition via spred. For each shape definition spred, we also declare a heap-independent invariant $\pi_{0}$ over the parameters $\left\{\operatorname{self}, v^{*}\right\}$ that holds for each instance of the predicate.

Each method meth and while loop is declared with preand post-conditions of the form $\Phi_{p r} * \Phi_{p o}$. For simplicity, we assume that variable names declared in each method are all distinct and that parameters are passed by-value. Primed notation is used to denote the latest value of variables and may appear in the postcondition of loops. For example, a simple loop with pre/post conditions is shown below: 


\begin{tabular}{|c|c|}
\hline$P$ & tdecl $::=$ datat $\mid$ spred \\
\hline datat & field $::=t v \quad t::=c \mid \tau$ \\
\hline & $::=$ int $\mid$ bool $\mid$ float | void \\
\hline spred & $::=c\left\langle v^{*}\right\rangle \equiv \Phi$ inv $\pi_{0}$ \\
\hline meth & $::=t m n\left((t v)^{*}\right)$ where $\bigwedge\left(\Phi_{p r} * \Phi_{p o}\right)^{*}\{e\}$ \\
\hline$e$ & $::=\operatorname{null}\left|k^{\tau}\right| v|v \cdot f| v:=e\left|v_{1} \cdot f:=v_{2}\right|$ new $c\left(v^{*}\right)$ \\
\hline & $\begin{array}{l}\left|e_{1} ; e_{2}\right| t v ; e\left|m n\left(v^{*}\right)\right| \text { if } v \text { then } e_{1} \text { else } e_{2} \\
\mid \text { while } v \text { where } \bigwedge\left(\Phi_{p r} * \Phi_{p o}\right)^{*} \text { do } e\end{array}$ \\
\hline$\Phi$ & $::=\bigvee\left(\exists v^{*} \cdot \kappa \wedge \pi\right)^{*} \quad \pi::=\gamma \wedge \phi$ \\
\hline$\gamma$ & $::=v_{1}=v_{2}|v=\operatorname{null}| v_{1} \neq v_{2}|v \neq \operatorname{null}| \gamma_{1} \wedge \gamma_{2}$ \\
\hline$\kappa$ & $::=\operatorname{emp}\left|v:: c\left\langle v^{*}\right\rangle\right| \kappa_{1} * \kappa_{2}$ \\
\hline$\Delta$ & $::=\Phi\left|\Delta_{1} \vee \Delta_{2}\right| \Delta \wedge \pi\left|\Delta_{1} * \Delta_{2}\right| \exists v \cdot \Delta$ \\
\hline$\phi$ & $::=\varphi|b| a\left|\phi_{1} \wedge \phi_{2}\right| \phi_{1} \vee \phi_{2}|\neg \phi| \exists v \cdot \phi \mid \forall v \cdot \phi$ \\
\hline$b$ & $::=$ true $\mid$ false $|v| b_{1}=b_{2}$ \\
\hline$a$ & $::=s_{1}=s_{2} \mid s_{1} \leq s_{2}$ \\
\hline$s$ & $\begin{aligned}::= & k^{\text {int }}|v| k^{\text {int }} \times s\left|s_{1}+s_{2}\right|-s \mid \max \left(s_{1}, s_{2}\right) \\
& \left|\min \left(s_{1}, s_{2}\right)\right||B|\end{aligned}$ \\
\hline$\varphi$ & $::=v \in B\left|B_{1}=B_{2}\right| B_{1} \sqsubset B_{2}|\forall v \in B \cdot \phi| \exists v \in B \cdot \phi$ \\
\hline$B$ & $::=B_{1} \sqcup B_{2}\left|B_{1} \sqcap B_{2}\right| B_{1}-B_{2}|\{\}|\{v\}$ \\
\hline
\end{tabular}

Figure 1. A Core Imperative Language

while $\mathrm{x}<0$ where true $*\left(\mathrm{x}>0 \wedge \mathrm{x}^{\prime}=\mathrm{x}\right) \vee\left(\mathrm{x} \leq 0 \wedge \mathrm{x}^{\prime}=0\right)$ do $\{\mathrm{x}:=\mathrm{x}+1\}$

Here $\mathrm{x}$ and $\mathrm{x}^{\prime}$ denote the values of variable $\mathrm{x}$ at the entry and exit of the loop, respectively.

The separation constraints we use are in a disjunctive normal form $\Phi$. Each disjunct consists of a $*$-separated heap constraint $\kappa$, referred to as heap part, and a heapindependent formula $\pi$, referred to as pure part. The pure part does not contain any heap nodes and is presently restricted to pointer equality/disequality $\gamma$, Presburger arithmetic $\phi$ and bag constraint $\varphi$. Furthermore, $\Delta$ denotes a composite formula that could always be normalised into the $\Phi$ form (see Figure 3). The semantic model for the separation constraints is left in the technical report [19].

Separation constraints are used in pre/post conditions and shape definitions. In order to handle them correctly without running into unmatched residual heap nodes, we require each separation constraint to be well-formed, as given by the following definitions:

Definition 3.1 (Accessible) A variable is said to be accessible w.r.t. a shape predicate if it is a parameter or it is a special variable, either self or res.

Definition 3.2 (Reachable) Given a heap constraint $\kappa=\mathrm{p}: \mathrm{c}\left\langle\mathrm{v}^{*}\right\rangle * \kappa_{1}$, node $\mathrm{p}: \mathrm{c}\left\langle\mathrm{v}^{*}\right\rangle$ is reachable from $a$ variable $\mathrm{q}$ if and only if the following relation holds:

$$
\begin{aligned}
& \operatorname{reach}\left(\kappa, \mathrm{q}, \mathrm{p}:: \mathrm{c}\left\langle\mathrm{v}^{*}\right\rangle\right)={ }_{d f}(\mathrm{p}=\mathrm{q}) \\
& \vee\left(\kappa_{1}=\mathrm{q}:: \mathrm{c}_{\mathrm{q}}\left\langle. ., \mathrm{r}, . . . * \kappa_{2} \wedge \operatorname{reach}\left(\kappa_{2}, \mathrm{r}, \mathrm{p}:: \mathrm{c}\left\langle\mathrm{v}^{*}\right\rangle\right)\right)\right.
\end{aligned}
$$

Definition 3.3 (Well-Formed Constraint) $A$ separation constraint $\Phi$ is well-formed if ( $i)$ every data node and shape predicate are reachable from their accessible variables, (ii) it is in a disjunctive normal form $\bigvee\left(\exists v^{*} \cdot \kappa \wedge \gamma \wedge \phi\right)^{*}$ where $\kappa$ is for heap nodes, $\gamma$ is for pointer constraint, and $\phi$ is for arithmetic formula.

The primary significance of the well-formed condition is that all heap nodes of a heap constraint are reachable from accessible variables. This allows the entailment checking procedure to correctly match nodes from the consequent with nodes from the antecedent of an entailment relation.

Arbitrary recursive shape relation can lead to nontermination in unfold/fold reasoning. To avoid that problem, we propose to use only well-founded shape predicates in our framework.

Definition 3.4 (Well-Founded Predicate) A shape predicate is said to be well-founded if it satisfies four conditions, namely: (i) it is a well-formed constraint, (ii) the parameter self may only be bound to a data node and not a predicate, (iii) only self is allowed to be bound to a data node and (iv) every predicate is reachable from self.

Note that the definitions above are syntactic and can easily be enforced. An example of well-founded shape predicates is avl - binary tree with near balanced heights, as follows :

$$
\begin{aligned}
& \operatorname{avl}\langle\mathrm{n}, \mathrm{h}\rangle \equiv(\operatorname{self}=\mathrm{null} \wedge \mathrm{n}=0 \wedge \mathrm{h}=0) \\
& \vee\left(\operatorname{self}:: \operatorname{node} 2\langle-, \mathrm{p}, \mathrm{q}\rangle * \mathrm{p}: \operatorname{avl}\left\langle\mathrm{n}_{1}, \mathrm{~h}_{1}\right\rangle * \mathrm{q}:: \operatorname{avl}\left\langle\mathrm{n}_{2}, \mathrm{~h}_{2}\right\rangle\right. \\
& \left.\wedge \mathrm{n}=1+\mathrm{n}_{1}+\mathrm{n}_{2} \wedge \mathrm{h}=1+\max \left(\mathrm{h}_{1}, \mathrm{~h}_{2}\right) \wedge-1 \leq \mathrm{h}_{1}-\mathrm{h}_{2} \leq 1\right) \\
& \quad \operatorname{inv} \mathrm{n}, \mathrm{h} \geq 0
\end{aligned}
$$

In contrast, the following three shape definitions are not well-founded.

$$
\begin{aligned}
& \text { foo }\langle\mathrm{n}\rangle \equiv \text { self::foo }\langle\mathrm{m}\rangle \wedge \mathrm{n}=\mathrm{m}+1 \\
& \text { goo }\langle\rangle \equiv \operatorname{self::\operatorname {node}\langle -}\left\langle_{-}\right\rangle * \mathrm{q}:: \operatorname{goo}\langle\rangle \\
& \text { too }\langle\rangle \equiv \text { self::node }\left\langle_{-}, \mathrm{q}\right\rangle * \mathrm{q}:: \text { node }\left\langle_{-},{ }_{-}\right\rangle
\end{aligned}
$$

For foo, the self identifier is bound to a shape predicate. For goo, the heap node pointed by q is not reachable from variable self. For too, an extra data node is bound to a non-self variable. The first example may cause infinite unfolding, while the second example captures an unreachable (junk) heap that cannot be located by our entailment procedure. The last example is just a syntactic restriction to facilitate termination of proof reasoning, and can be easily overcome by introducing intermediate predicates.

\subsection{Forward Verification}

We use $P$ to denote the program being checked. With pre/post conditions declared for each method in $P$, we can 


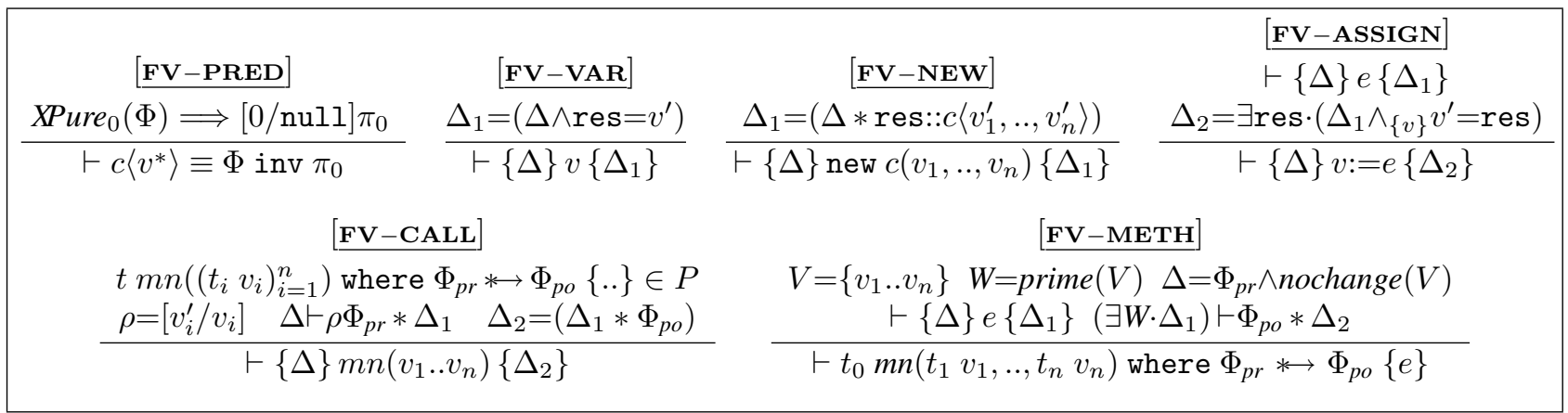

Figure 2. Some Forward Verification Rules

apply modular verification to a method's body using Hoarestyle triples $\vdash\left\{\Delta_{1}\right\}$ e $\left\{\Delta_{2}\right\}$. These are forward verification rules that expect $\Delta_{1}$ to be given before computing $\Delta_{2}$. Some rules are given in Fig 2 while others are left in the technical report [19]. They are used to track heap states as accurately as possible with path-, flow-, and contextsensitivity. For each call site, [FV-CALL] ensures that the callee's precondition is satisfied. For each method definition, [FV-METH] checks that its postcondition holds for the method body assuming its precondition. A method postcondition may capture only part of the heap at the end of the method, leaving the residue heap nodes in $\Delta_{2}$. For each shape definition, [FV-PRED] checks that its given invariant is a consequence of the well-founded heap formula. The soundness of the forward verification is also left in the technical report.

We now explain the operators/functions used in our verification rules. The operator $\wedge_{\{v\}}$ used in the assignment rule is an instance of composition with update operators. Given a state $\Delta_{1}$, a state change $\Delta_{2}$, and a set of variables to be updated $X=\left\{x_{1}, \ldots, x_{n}\right\}$, the composition operator $\oplus_{X}$ is defined as:

$$
\begin{aligned}
& \Delta_{1} \oplus_{X} \Delta_{2}={ }_{d f} \exists r_{1} . . r_{n} \cdot \rho_{1} \Delta_{1} \oplus \rho_{2} \Delta_{2} \\
& \text { where } r_{1}, \ldots, r_{n} \text { are fresh variables; } \\
& \rho_{1}=\left[r_{i} / x_{i}^{\prime}\right]_{i=1}^{n} ; \rho_{2}=\left[r_{i} / x_{i}\right]_{i=1}^{n}
\end{aligned}
$$

Note that $\rho_{1}$ and $\rho_{2}$ are substitutions that link each latest value of $x_{i}^{\prime}$ in $\Delta_{1}$ with the corresponding initial value $x_{i}$ in $\Delta_{2}$ via a fresh variable $r_{i}$. The binary operator $\oplus$ is either $\wedge$ or $*$. The function nochange $(V)$ returns a formula asserting that the unprimed and primed versions of each variable in $V$ are equal; prime $(V)$ returns the primed form of all variables in $V . \quad\left[e^{*} / v^{*}\right]$ represents substitutions of $v^{*}$ by $e^{*}$. A special case is $[0 / \mathrm{null}]$, which denotes replacement of null by 0 . Normalization rules for separation constraints are given in Figure 3. XPure is described in the next section.

\subsection{Forward Verification Example}

We present the detailed verification of the first branch of the insert function from $\mathrm{Sec} 2$. Note that program variables appear primed in formulae whereas logical variables unprimed. The proof is straightforward, except for the last step where a disjunctive heap state is folded to form a shape predicate. The procedure to perform the folding step is presented in Sec 4. $\left\{\mathrm{x}^{\prime}:: \operatorname{sortl}\langle\mathrm{n}, \mathrm{mi}, \mathrm{ma}\rangle * \mathrm{vn}^{\prime}:: \operatorname{node}\left\langle\mathrm{v},{ }_{-}\right\rangle\right\} / /$precondition

if $(\mathbf{v n} . v a l \leq \mathrm{x} . \mathrm{val})$ then \{

$\left\{\left(\mathrm{x}^{\prime}:\right.\right.$ node $\langle\mathrm{mi}, \mathrm{null}\rangle * \mathrm{vn}^{\prime}::$ node $\left\langle\mathrm{v},{ }_{-}\right\rangle \wedge \mathrm{mi}=\operatorname{ma} \wedge \mathrm{n}=1$

$\wedge \mathrm{v} \leq \mathrm{mi}) \vee\left(\exists \mathrm{q}, \mathrm{k} \cdot \mathrm{x}^{\prime}:: \operatorname{node}\langle\mathrm{mi}, \mathrm{q}\rangle * \mathrm{q}:: \operatorname{sortl}\langle\mathrm{n}-1, \mathrm{k}, \mathrm{ma}\rangle\right.$

$\left.\left.* \mathrm{vn}^{\prime}:: \operatorname{node}\langle\mathrm{v},-\rangle \wedge \mathrm{mi} \leq \mathrm{k} \wedge \mathrm{mi} \leq \mathrm{ma} \wedge \mathrm{n} \geq 2 \wedge \mathrm{v} \leq \mathrm{mi}\right)\right\}$

// unfold and conditional

vn.next : $=\mathbf{x}$

$\left\{\left(\mathrm{x}^{\prime}:\right.\right.$ :node $\langle\mathrm{mi}, \mathrm{null}\rangle * \mathrm{vn}^{\prime}::$ node $\left\langle\mathrm{v}, \mathrm{x}^{\prime}\right\rangle \wedge \mathrm{mi}=\mathrm{ma} \wedge \mathrm{n}=1$

$\wedge \mathrm{v} \leq \mathrm{mi}) \vee\left(\exists \mathrm{q}, \mathrm{k} \cdot \mathrm{x}^{\prime}::\right.$ node $\langle\mathrm{mi}, \mathrm{q}\rangle * \mathrm{q}:: \operatorname{sortl}\langle\mathrm{n}-1, \mathrm{k}, \mathrm{ma}\rangle$

$\left.\left.* \mathrm{vn}^{\prime}:: \operatorname{node}\left\langle\mathrm{v}, \mathrm{x}^{\prime}\right\rangle \wedge \mathrm{mi} \leq \mathrm{k} \wedge \mathrm{mi} \leq \mathrm{ma} \wedge \mathrm{n} \geq 2 \wedge \mathrm{v} \leq \mathrm{mi}\right)\right\}$

\section{$/ /$ field update}

vn

$\left\{\left(\mathrm{x}^{\prime}::\right.\right.$ node $\langle\mathrm{mi}, \mathrm{null}\rangle * \mathrm{vn}^{\prime}:: \operatorname{node}\left\langle\mathrm{v}, \mathrm{x}^{\prime}\right\rangle \wedge \mathrm{mi}=\mathrm{ma} \wedge \mathrm{n}=1$

$\wedge \mathrm{v} \leq \mathrm{mi} \wedge$ res $\left.=\mathrm{vn}^{\prime}\right) \vee\left(\exists \mathrm{q}, \mathrm{k} \cdot \mathrm{x}^{\prime}::\right.$ node $\langle\mathrm{mi}, \mathrm{q}\rangle$

$* \mathrm{q}:: \operatorname{sortl}\langle\mathrm{n}-1, \mathrm{k}, \mathrm{ma}\rangle * \mathrm{vn}^{\prime}:$ node $\left\langle\mathrm{v}, \mathrm{x}^{\prime}\right\rangle \wedge \mathrm{mi} \leq \mathrm{k} \wedge \mathrm{mi} \leq \mathrm{ma}$

$\left.\left.\wedge \mathrm{n} \geq 2 \wedge \mathrm{v} \leq \mathrm{mi} \wedge \mathrm{res}=\mathrm{vn}^{\prime}\right)\right\} / /$ returned value

\}

$\{$ res::sortl $\langle\mathrm{n}+1, \min (\mathrm{v}, \mathrm{mi}), \max (\mathrm{v}, \mathrm{ma})\rangle\}$

$/ /$ fold to postcondition

\section{Entailment}

We present in this section the entailment checking rules for the class of constraints used by our verification system.

\subsection{Separation Constraint Approximation}

Entailment between separation formulae (detailed in section 4.2) is reduced to entailment between pure formulae by successively removing heap nodes from the consequent until only a pure formula remains. When the consequent is 


\begin{tabular}{|llll|}
$\left(\Delta_{1} \vee \Delta_{2}\right) \wedge \pi$ & $\leadsto\left(\Delta_{1} \wedge \pi\right) \vee\left(\Delta_{2} \wedge \pi\right)$ & $\left(\gamma_{1} \wedge \phi_{1}\right) \wedge\left(\gamma_{2} \wedge \phi_{2}\right)$ & $\sim\left(\gamma_{1} \wedge \gamma_{2}\right) \wedge\left(\phi_{1} \wedge \phi_{2}\right)$ \\
$\left(\Delta_{1} \vee \Delta_{2}\right) * \Delta$ & $\leadsto\left(\Delta_{1} * \Delta\right) \vee\left(\Delta_{2} * \Delta\right)$ & $(\exists x \cdot \Delta) \wedge \pi$ & $\sim \exists y \cdot([y / x] \Delta \wedge)$ \\
$\left(\kappa_{1} \wedge \pi_{1}\right) *\left(\kappa_{2} \wedge \pi_{2}\right)$ & $\leadsto\left(\kappa_{1} * \kappa_{2}\right) \wedge\left(\pi_{1} \wedge \pi_{2}\right)$ & $\left(\exists x \cdot \Delta_{1}\right) * \Delta_{2}$ & $\leadsto \exists y \cdot\left([y / x] \Delta_{1} * \Delta_{2}\right)$ \\
$\left(\kappa_{1} \wedge \pi_{1}\right) \wedge\left(\pi_{2}\right)$ & $\leadsto \kappa_{1} \wedge\left(\pi_{1} \wedge \pi_{2}\right)$ & & \\
\hline
\end{tabular}

Figure 3. Normalization Rules

pure, the heap formula in the antecedent is soundly approximated by function XPure ${ }_{n}$. The function $X$ Pure $_{n}(\Phi)$, whose definition is given in Fig 4, returns a sound approximation of $\Phi$ as formula ex $i^{*} \cdot \bigvee\left(\exists v^{*} \cdot \pi\right)^{*}$ where $i^{*}$ are (nonnull) distinct symbolic addresses of heap nodes of $\Phi$. The function IsData $(c)$ returns true if $c$ is a data node, while IsPred $(c)$ returns true if $c$ is a shape predicate.

We illustrate how these functions work by computing XPure $_{1}(\mathrm{p}:: 11\langle\mathrm{n}\rangle)$. Let $\Phi$ be the body of the 11 predicate.

$$
\begin{aligned}
& \operatorname{Inv_{0}}(\mathrm{p}:: 1 \mathrm{l}\langle\mathrm{n}\rangle)=_{d f} \mathrm{n} \geq 0 \\
& X_{\text {Pure }_{0}}(\Phi)=_{d f} \operatorname{ex~} \mathrm{j} \cdot(\operatorname{self}=0 \wedge \mathrm{n}=0) \\
& \vee(\operatorname{self}=j \wedge j>0 \wedge n-1 \geq 0) \\
& \operatorname{Inv}_{1}(\mathrm{p}:: 1 \mathrm{l}\langle\mathrm{n}\rangle)={ }_{d f}[\mathrm{p} / \mathrm{self}] X \text { ure }_{0}(\Phi) \\
& X \text { Pure }_{1}(\mathrm{p}:: 1 \mathrm{l}\langle\mathrm{n}\rangle)=_{d f} \operatorname{exi} \cdot[\mathrm{i} / \mathrm{j}] \operatorname{Inv}_{1}(\mathrm{p}:: \mathrm{ll}\langle\mathrm{n}\rangle) \\
& \leadsto \exists \mathrm{i} \cdot(\mathrm{p}=0 \wedge \mathrm{n}=0) \vee(\mathrm{p}=\mathrm{i} \wedge \mathrm{i}>0 \wedge \mathrm{n}>0) \\
& =(\mathrm{p}=0 \wedge \mathrm{n}=0) \vee(\mathrm{p}>0 \wedge \mathrm{n}>0)
\end{aligned}
$$

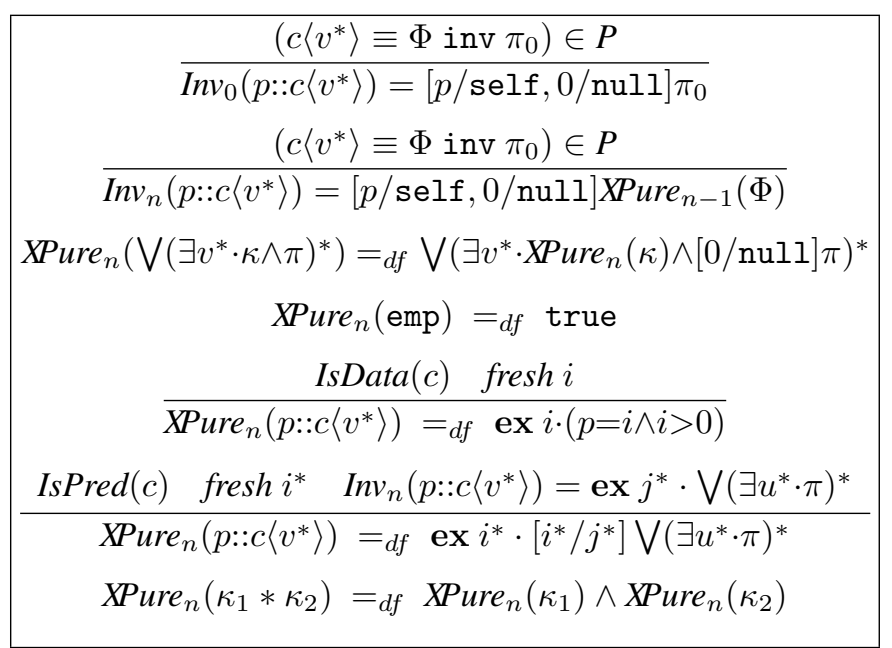

Figure 4. XPure: Translating to Pure Form

The following normalization rules are also used :

$$
\begin{array}{ll}
\left(\operatorname{ex} I \cdot \phi_{1}\right) \vee\left(\operatorname{ex} J \cdot \phi_{2}\right) & \leadsto \operatorname{ex} I \cup J \cdot\left(\phi_{1} \vee \phi_{2}\right) \\
\exists v \cdot(\operatorname{ex} I \cdot \phi) & \leadsto \operatorname{ex} I \cdot(\exists v \cdot \phi) \\
\left(\operatorname{ex} I \cdot \phi_{1}\right) \wedge\left(\operatorname{ex} J \cdot \phi_{2}\right) & \leadsto \operatorname{ex} I \cup J \cdot \phi_{1} \wedge \phi_{2} \wedge \bigwedge_{i \in I, j \in J} i \neq j
\end{array}
$$

The ex $i^{*}$ construct is converted to $\exists i^{*}$ when the formula is used as a pure formula. The soundness of XPure ${ }_{n}$ is formalized by :

Lemma 4.1 (Sound Invariant) Given a shape predicate $c\left\langle v^{*}\right\rangle \equiv \Phi$ inv $\pi_{0}$, we have $\Phi \models \operatorname{Inv}_{n}\left(\operatorname{self}:: c\left\langle v^{*}\right\rangle\right)$ if $X$ Pure $_{0}(\Phi) \Longrightarrow[0 /$ null $] \pi_{0} . \pi_{0}$ is said to be sound.

Proof: By structural induction on $\Phi$.

Lemma 4.2 (Sound Abstraction) Given a separation constraint $\Phi$ where the invariants of the predicates appearing in $\Phi$ are sound, we have $\Phi \models X$ Pure $_{n}(\Phi)$.

Proof : By structural induction on $\Phi$.

Lemma 4.1 ensures that a supplied invariant that passes [FV-PRED] is a semantic consequence of the predicate. Lemma 4.2 asserts that it is safe to approximate an antecedent by using XPure if all the predicate invariants are sound. They also allow the possibility of obtaining a more precise invariant by applying XPure one or more times. For example, when given a pure invariant $\mathrm{n} \geq 0$ for the predicate $l l\langle\mathrm{n}\rangle$, a single application returns ex $i \cdot($ self $=0 \wedge \mathrm{n}=0 \vee$ self $=i \wedge i>0 \wedge n>0$ ) which is sound and more precise, as it relates the nullness of the self pointer with the size $n$ of the list.

The invariants associated with shape predicates play an important role in our system. Without the knowledge $m \geq 0$, the entailment $\mathrm{x}::$ node $\langle,, \mathrm{y}\rangle * \mathrm{y}:: 1 \mathrm{l}\langle\mathrm{m}\rangle \vdash \mathrm{x}:: 1 \mathrm{l}\langle\mathrm{n}\rangle \wedge \mathrm{n} \geq 1$ would not have succeeded due to $n \geq 1$. Without the more precise derived invariant using $X P$ ure for predicate 11 , the entailment $\mathrm{x}:: 1 \mathrm{l}\langle\mathrm{n}\rangle \wedge \mathrm{n}>0 \vdash \mathrm{x} \neq \mathrm{null}$ would not have succeeded either.

\subsection{Separation Constraint Entailment}

We express the main procedure for heap entailment by the relation

$$
\Delta_{A} \vdash_{V}^{\kappa} \Delta_{C} * \Delta_{R}
$$

which denotes $\kappa * \Delta_{A} \vdash \exists V \cdot\left(\kappa * \Delta_{C}\right) * \Delta_{R}$.

The purpose of heap entailment is to check that heap nodes in the antecedent $\Delta_{A}$ are sufficiently precise to cover all nodes from the consequent $\Delta_{C}$, and to compute a residual heap state $\Delta_{R} . \kappa$ is the history of nodes from the antecedent that have been used to match nodes from the consequent, $V$ is the list of existentially quantified variables from the consequent. Note that $k$ and $V$ are derived. The entailment checking procedure is invoked with $\kappa=$ emp and $V=\emptyset$. The entailment checking rules are given in Fig 5 . We discuss the matching rule in what follows, and leave unfold/fold rules to Sec 5 .

The procedure works by successively matching up heap nodes that can be proven aliased. As the matching process is incremental, we keep the successfully matched nodes from 


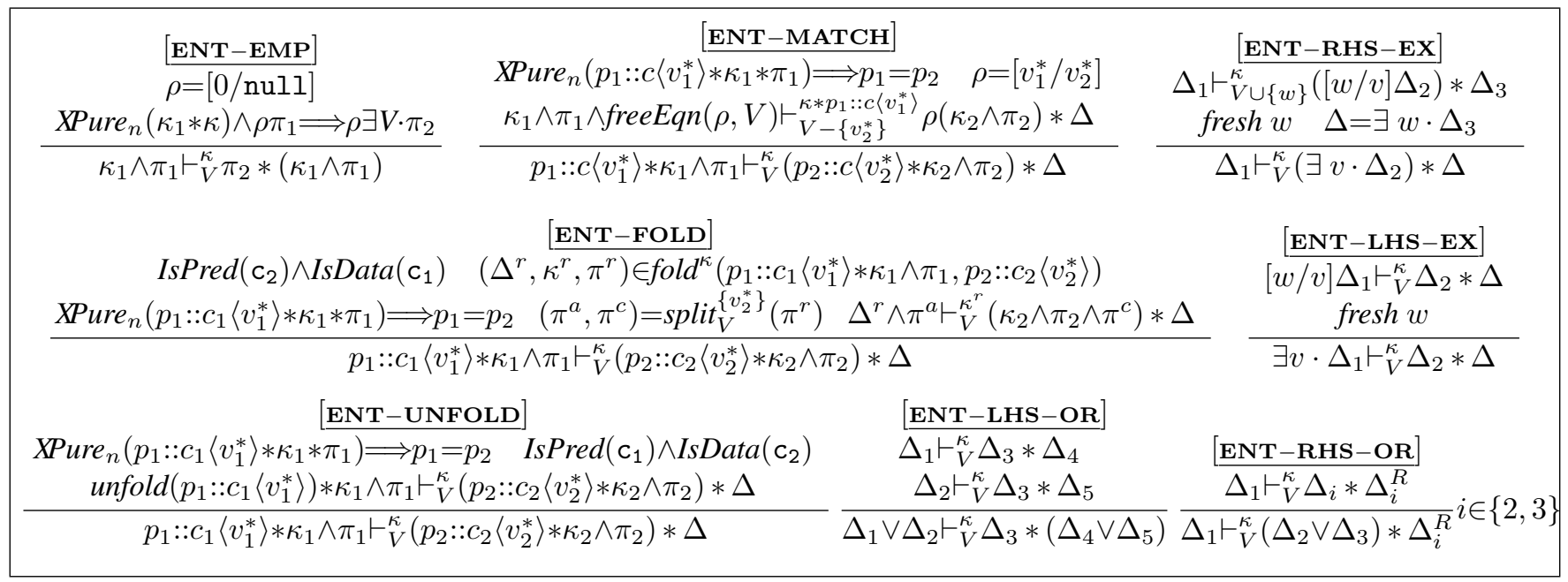

Figure 5. Separation Constraint Entailment

antecedent in $\kappa$ for better precision. For example, consider the following (valid) proof:

$$
\begin{gathered}
(((\mathrm{p}=\mathrm{null} \wedge \mathrm{n}=0) \vee(\mathrm{p} \neq \mathrm{null} \wedge \mathrm{n}>0)) \\
\wedge \mathrm{n}>0 \wedge \mathrm{m}=\mathrm{n}) \Longrightarrow \mathrm{p} \neq \mathrm{null} \\
\Delta_{\mathrm{R}}=(\mathrm{n}>0 \wedge \mathrm{m}=\mathrm{n}) \\
\frac{\mathrm{n}>0 \wedge \mathrm{m}=\mathrm{n} \vdash^{\mathrm{p}:: 11\langle\mathrm{n}\rangle} \mathrm{p} \neq \mathrm{null} * \Delta_{\mathrm{R}}}{\mathrm{p}:: \mathrm{ll}\langle\mathrm{n}\rangle \wedge \mathrm{n}>0 \vdash \mathrm{p}:: 1 \mathrm{~m}\langle\mathrm{~m}\rangle \wedge \mathrm{p} \neq \mathrm{null} * \Delta_{\mathrm{R}}}
\end{gathered}
$$

Had the predicate $\mathrm{p}:: 11\langle\mathrm{n}\rangle$ not been kept and used, the proof would not have succeeded. Such an entailment would be useful when, for example, a list with positive length $n$ is used as input for a function that requires a non-empty list.

Another feature of the entailment procedure is exemplified by the transfer of $m=n$ to the antecedent (and subsequently to the residue). In general, when a match occurs (rule [ENT-MATCH] ) and an argument of the heap node coming from the consequent is free, the entailment procedure binds the argument to the corresponding variable from the antecedent and moves the equality to the antecedent. In our system, free variables in consequent are variables from method preconditions. Hence these bindings act as substitutions that have to be kept in antecedent to allow subsequent program state (from residual heap) to be aware of their values. This process is formalized by the function freeEqn below, where $V$ is the set of existentially quantified variables:

$$
\begin{aligned}
& \text { freeEqn }\left(\left[u_{i} / v_{i}\right]_{i=1}^{n}, V\right)={ }_{d f} \\
& \quad \text { let } \pi_{i}=\left(\text { if } v_{i} \in V \text { then true else } v_{i}=u_{i}\right) \text { in } \bigwedge_{i=1}^{n} \pi_{i}
\end{aligned}
$$

For soundness, we perform a preprocessing step to ensure that variables appearing as arguments of heap nodes and predicates are i) distinct and ii) if they are free, they do not appear in the antecedent by adding (existentially quantified) fresh variables and equalities. This guarantees that the generated substitutions are well-defined. It also guar- antees that the formula generated by freeEqn does not introduce any additional constraints over existing variables in the antecedent, as one side of each equation does not appear anywhere else in the antecedent.

\section{Unfold/Fold Mechanism}

Unfold/fold operations can be used to handle wellfounded inductive predicates in a deductive manner. In particular, we can unfold a predicate that appears in the antecedent if it matches with a data node in the consequent. Correspondingly, we fold a predicate that appears in the consequent if it matches with a data node in the antecedent. The well-founded condition is sufficient to ensure termination.

\subsection{Unfolding a Shape Predicate in the Antecedent}

We apply an unfold operation on a predicate in the antecedent that matches with a data node in the consequent. Consider :

$$
\begin{aligned}
\mathrm{x}:: 113\langle\mathrm{n}, \mathrm{B}\rangle \wedge \mathrm{n}>2 \vdash \quad & (\exists \mathrm{r} \cdot \mathrm{x}:: \operatorname{node}\langle\mathrm{r}, \mathrm{y}\rangle \wedge \mathrm{y} \neq \mathrm{null} \\
& \wedge \mathrm{r} \in \mathrm{B}) * \Delta_{\mathrm{R}}
\end{aligned}
$$

where $\Delta_{R}$ captures the residue of entailment. For the entailment to succeed, we would first unfold the $113\langle$ n, B $\rangle$ predicate in the antecedent:

$$
\begin{aligned}
& \exists \mathrm{q}_{1}, \mathrm{v} \cdot \mathrm{x}: \mathrm{node}\left\langle\mathrm{v}, \mathrm{q}_{1}\right\rangle * \mathrm{q}_{1}:: 113\left\langle\mathrm{n}-1, \mathrm{~B}_{1}\right\rangle \wedge \mathrm{n}>2 \\
& \wedge \mathrm{B}=\mathrm{B}_{1} \cup\{\mathrm{v}\} \vdash(\exists \mathrm{r} \cdot \mathrm{x}: \mathrm{node}\langle\mathrm{r}, \mathrm{y}\rangle \wedge \mathrm{y} \neq \mathrm{null} \wedge \mathrm{r} \in \mathrm{B}) * \Delta_{\mathrm{R}}
\end{aligned}
$$

After removing the existential quantifiers, we obtain:

$$
\begin{aligned}
& \mathrm{x}:: \text { node }\left\langle\mathrm{v}, \mathrm{q}_{1}\right\rangle * \mathrm{q}_{1}:: 113\left\langle\mathrm{n}-1, \mathrm{~B}_{1}\right\rangle \wedge \mathrm{n}>2 \wedge \mathrm{B}=\mathrm{B}_{1} \cup\{\mathrm{v}\} \\
& \quad \vdash(\mathrm{x}:: \operatorname{node}\langle\mathrm{r}, \mathrm{y}\rangle \wedge \mathrm{y} \neq \mathrm{null} \wedge \mathrm{r} \in \mathrm{B}) * \Delta_{\mathrm{R}}
\end{aligned}
$$


The data node in the consequent is then matched up, giving:

$$
\begin{aligned}
& \mathrm{q}_{1}:: 113\left\langle\mathrm{n}-1, \mathrm{~B}_{1}>\wedge \mathrm{n}>2 \wedge \mathrm{B}=\mathrm{B}_{1} \cup\{\mathrm{v}\} \wedge \mathrm{q}_{1}=\mathrm{y}\right. \\
& \quad \vdash\left(\mathrm{q}_{1} \neq \text { null } \wedge \mathrm{v} \in \mathrm{B}\right) * \Delta_{\mathrm{R}}
\end{aligned}
$$

Due to the well-founded condition, each unfolding exposes a data node that matches the data node in the consequent. Thus a reduction of the consequent immediately follows, which contributes to the termination of the entailment check. A formal definition of unfolding is given by the [UNFOLDING] rule:

$$
\frac{\left[\frac{\text { UNFOLDING }}{c\left\langle v^{*}\right\rangle \equiv \Phi \in P}\right.}{\operatorname{unfold}\left(p:: c\left\langle v^{*}\right\rangle\right)=_{d f}[p / \text { self }] \Phi}
$$

\subsection{Folding a Shape Predicate in the Con- sequent}

We apply a fold operation when a data node in the antecedent matches with a predicate in the consequent. An example is :

$$
\begin{gathered}
\mathrm{x}: \text { node }\left\langle 1, \mathrm{q}_{1}\right\rangle * \mathrm{q}_{1}:: \text { node }\langle 2, \text { null }\rangle * \mathrm{y}:: \text { node }\langle 3, \text { null }\rangle \\
\vdash(\mathrm{x}:: 113\langle\mathrm{n}, \mathrm{B}\rangle \wedge \mathrm{n}>1 \wedge 1 \in \mathrm{B}) * \Delta_{\mathrm{R}}
\end{gathered}
$$

The fold step may be recursively applied but is guaranteed to terminate for well-founded predicate as it will reduce a data node in the antecedent for each recursive invocation. This reduction in the antecedent cannot go on forever. Furthermore, the fold operation may introduce bindings for the parameters of the folded predicate. In the above, we obtain $\exists \mathrm{n}_{1}, \mathrm{n}_{2} \cdot \mathrm{n}=\mathrm{n}_{1}+1 \wedge \mathrm{n}_{1}=\mathrm{n}_{2}+1 \wedge \mathrm{n}_{2}=0$ and $\exists B_{1}, B_{2} \cdot B=B_{1} \cup\{2\} \wedge B_{1}=\{1\} \cup B_{2} \wedge B_{2}=\{\}$, where $\mathrm{n}_{1}, \mathrm{n}_{2}, \mathrm{~B}_{1}, \mathrm{~B}_{2}$ are existential variables introduced by the folding process, and are subsequently eliminated. Each of the previous formulae may be transferred to the antecedent if $\mathrm{n}$ and $\mathrm{B}$ are free, respectively. Otherwise, they will be kept in the consequent. Since $\mathrm{n}$ and B are indeed free, our folding step would finally derive:

$$
\begin{aligned}
& \mathrm{y}:: \text { node }\langle 3, \text { null }\rangle \wedge \mathrm{n}=2 \wedge \mathrm{B}=\{1,2\} \\
& \quad \vdash(\mathrm{n}>1 \wedge 1 \in \mathrm{B}) * \Delta_{\mathrm{R}}
\end{aligned}
$$

The effects of folding may seem similar to unfolding the predicate in the consequent. However, there is a subtle difference in their handling of bindings for free derived variables. If we choose to use unfolding on the consequent instead, these bindings may not be transferred to the antecedent. Consider the example below where $\mathrm{n}$ is free :

$$
\mathrm{z}=\mathrm{null} \vdash \mathrm{z}:: 113\langle\mathrm{n}, \mathrm{B}\rangle \wedge \mathrm{n}>-1 * \Delta_{\mathrm{R}}
$$

By unfolding the predicate $11\langle\mathrm{n}\rangle$ in the consequent, we obtain :

$$
\begin{aligned}
\mathrm{z}=\mathrm{null} & \vdash(\mathrm{z}=\mathrm{null} \wedge \mathrm{n}=0 \wedge \mathrm{B}=\{\} \wedge \mathrm{n}>-1) \\
& \vee\left(\exists \mathrm{q}, \mathrm{v} \cdot \mathrm{z}:: \text { node }\langle\mathrm{v}, \mathrm{q}\rangle * \mathrm{q}:: 113\left\langle\mathrm{n}-1, \mathrm{~B}_{1}\right\rangle\right. \\
& \left.\wedge \mathrm{B}=\mathrm{B}_{1} \cup\{\mathrm{v}\} \wedge \mathrm{n}>-1\right) * \Delta_{\mathrm{R}}
\end{aligned}
$$

There are now two disjuncts in the consequent. The second one fails because it mismatches. The first one matches but still fails as the derived binding $\mathrm{n}=0$ was not transferred to the antecedent.

When a fold to a predicate $p_{2}:: c_{2}\left\langle v_{2}^{*}\right\rangle$ is performed, the constraints related to variables $v_{2}^{*}$ are important. The split function projects these constraints out and differentiates those constraints based on free variables.

$$
\begin{aligned}
& \operatorname{split}_{V}^{\left\{v_{2}^{*}\right\}}\left(\bigwedge_{i=1}^{n} \pi_{i}^{r}\right)= \\
& \text { let } \pi_{i}^{a}, \pi_{i}^{c}=\operatorname{if} F V\left(\pi_{i}^{r}\right) \cap v_{2}^{*}=\emptyset \text { then }(\text { true }, \text { true }) \\
& \quad \text { else if } F V\left(\pi_{i}^{r}\right) \cap V=\emptyset \text { then }\left(\pi_{i}^{r}, \text { true }\right) \\
& \quad \text { else }\left(\text { true }, \pi_{i}^{r}\right) \\
& \text { in }\left(\bigwedge_{i=1}^{n} \pi_{i}^{a}, \bigwedge_{i=1}^{n} \pi_{i}^{c}\right)
\end{aligned}
$$

$$
\begin{aligned}
& \text { [FOLDING] } \\
& c\left\langle v^{*}\right\rangle \equiv \Phi \in P \quad W_{i}=V_{i}-\left\{v^{*}, p\right\} \\
& \frac{\kappa \wedge \pi \vdash_{\left\{p, v^{*}\right\}}^{\kappa^{\prime}}[p / \text { self }] \Phi *\left\{\left(\Delta_{i}, \kappa_{i}, V_{i}, \pi_{i}\right)\right\}_{i=1}^{n}}{\operatorname{fold}^{\kappa^{\prime}}\left(\kappa \wedge \pi, p:: c\left\langle v^{*}\right\rangle\right)={ }_{d f}\left\{\left(\Delta_{i}, \kappa_{i}, \exists W_{i} \cdot \pi_{i}\right)\right\}_{i=1}^{n}}
\end{aligned}
$$

A formal definition of folding is specified by rule [FOLDING]. Some heap nodes from $\kappa$ are removed by the entailment procedure so as to match with the heap formula of predicate $p:: c\left\langle v^{*}\right\rangle$. This requires a special version of entailment that returns three extra things: (i) consumed heap nodes, (ii) existential variables used, and (iii) final consequent. The final consequent is used to return a constraint for $\left\{v^{*}\right\}$ via $\exists W_{i} \cdot \pi_{i}$. A set of answers is returned by the fold step as we allow it to explore multiple ways of matching up with its disjunctive heap state. Our entailment also handles empty predicates correctly with a couple of specialised rules.

\section{Soundness of Entailment}

The following theorems state that our entailment check procedure (given in Fig. 5) is sound and terminating. Proofs are given in the technical report [19].

Theorem 6.1 (Soundness) If entailment check

$\Delta_{1} \vdash \Delta_{2} * \Delta$ succeeds, we have: for all $s, h$, if $s, h \models \Delta_{1}$ then $s, h \models \Delta_{2} * \Delta$.

Theorem 6.2 (Termination) The entailment check $\Delta_{1} \vdash \Delta_{2} * \Delta$ always terminates.

\section{Implementation}

We have built a prototype system using Objective Caml. The proof obligations generated by our verification are discharged using either a constraint solver or a theorem prover. This is organised as an option in our system and currently 
covers automatic provers of Omega Calculator [22], Isabelle [21], and MONA [13].

Figure 6 summarizes a suite of programs tested. These examples use complicated recursion and data structures with sophisticated shape, size and bag properties. They show that our approach is general enough to handle interesting data structures such as sorted lists, sorted trees, priority queues, balanced trees, etc. in a uniform way. Verification time of a function includes time to verify all functions that it calls.

Arithmetic Constraints. The time required for shape and size verification is mostly within a couple of seconds when using the Omega Calculator to discharge the proof obligations (third column). In order to have a reference point for the Omega timings, we tried solving the same constraints with two other theorem provers : Isabelle (fourth column) and MONA (fifth column). From the former, we only used an automatic but incomplete prover, which caused some proofs to fail. The latter is an implementation of the weak monadic second-order logics WS1S and WS2S. Therefore, first-order variables can be compared and subjected only to addition with constants. As Presburger arithmetic allows the addition of arbitrary linear arithmetic terms, we converted its formulas into WS1S by encoding naturals as Base-2 bit strings. MONA translates WS1S and WS2S formulas into minimum DFAs (Deterministic Finite Automata) and GTAs (Guided Tree Automata), respectively. However, this translation may cause a state-space explosion. In our case, we confronted such a problem when verifying the quick_sort method, where the size of its corresponding automaton exceeded the available space.

Bag/Set Constraints. Bag constraints were solved using the multiset theory of Isabelle (sixth column), while weak monadic second-order theory of 1 successor WS1S from MONA was used to handle set constraints (seventh column). Due to the incompletness of the automatic prover that we used from Isabelle, some of the proofs failed (quick_sort, delete_max from priority queue heap and insert into a red-black tree). On the other hand, MONA seemed to provide satisfactory results when handling set constraints.

From our experiments, we may conclude that verification process is dominated by entailment proving of pure formulas that are fast with specialised solvers, such as Omega for Presburger constraint and MONA for set constraints. The timings for verifying shapes only (without size/bag proving) are benign, as reflected in the second column. An important future work is to design a combination strategy that allows specialised solvers to work well with mixed constraints and to handle large programs. We expect code modularity, decomposed shape views and multi-core parallelism to be important techniques in performance engineering of automated verification system.

\section{Related Work}

Separation Logic. The general framework of separation logic $[23,11]$ is highly expressive but undecidable. Likewise, [17] formalised the proof rules for handling abstract predicates (with scopes on visibility of predicates) but provided no automated procedure for checking the user supplied specifications. In the search for a decidable fragment of separation logic for automated verification, Berdine et al. [1] supports only a limited set of predicates without size properties, disjunctions and existential quantifiers. Similarly, Jia and Walker [12] postponed the handling of recursive predicates in their recent work on automated reasoning of pointer programs. Our approach is more pragmatic as we aim for a sound and terminating formulation of automated verification via separation logic but do not aim for completeness in the expressive fragment that we handle. On the inference front, Lee et al. [16] has conducted an intraprocedural analysis for loop invariants using grammar approximation under separation logic. Their analysis can handle a wide range of shape predicates with local sharing but is restricted to predicates with two parameters and without size properties. A recent work [9] has also formulated interprocedural shape inference but is restricted to just the list segment shape predicate. Sims [26] extends separation logic with fixpoint connectives and postponed substitution to express recursively defined formulae to model the analysis of while-loops. However, it is unclear how to check for entailment in their extended separation logic. While our work does not address the inference/analysis challenge, we have succeeded in providing direct support for automated verification via an expressive shape and size specification mechanism.

Shape Checking/Analysis. Many formalisms for shape analysis have been proposed for checking user programs' intricate manipulations of shapely data structures. One well-known work is Pointer Assertion Logic [18] by Moeller and Schwartzbach where shape specifications in monadic second-order logic are given by programmers for loop invariants and method pre/post conditions, and checked by their MONA tool. For shape inference, Sagiv et al. [25] presented a parameterised framework, called TVLA, using 3-valued logic formulae and abstract interpretation. Based on the properties expected of data structures, programmers must supply a set of predicates to the framework which are then used to analyse that certain shape invariants are maintained. However, most of these techniques were focused on analysing shape invariants, and did not attempt to track the size properties of complex data structures. An exception is the quantitative shape analysis of Rugina [24] where a data flow analysis was proposed to compute quantitative information for programs with destructive updates. By tracking unique points-to reference and its height 


\begin{tabular}{|c|c|c|c|c|c|c|}
\hline Programs & $\begin{array}{l}\text { Without } \\
\text { size/bag }\end{array}$ & $\begin{array}{c}\text { Omega } \\
\text { Calculator }\end{array}$ & $\begin{array}{l}\text { Isabelle } \\
\text { Prover }\end{array}$ & $\begin{array}{l}\text { MONA } \\
\text { Prover }\end{array}$ & $\begin{array}{l}\text { Isabelle } \\
\text { Prover }\end{array}$ & $\begin{array}{l}\text { MONA } \\
\text { Prover }\end{array}$ \\
\hline Linked List & & \multicolumn{3}{|c|}{ "verifies size/length } & \multicolumn{2}{|l|}{ verifies bag/set } \\
\hline delete & 0.02 & 0.09 & 8.35 & 0.33 & 5.00 & 0.34 \\
\hline reverse & 0.02 & 0.07 & 3.28 & 0.21 & 3.01 & 0.20 \\
\hline Circular Linked List & & \multicolumn{3}{|c|}{ verifies size + cyclic structure } & \multicolumn{2}{|c|}{ verifies bag/set + cyclic structure } \\
\hline delete (first) & 0.01 & 0.09 & 5.46 & 0.26 & 7.17 & 0.40 \\
\hline count & 0.04 & 0.16 & 14.99 & 0.71 & 21.01 & 2.29 \\
\hline Doubly Linked List & & \multicolumn{3}{|c|}{ verifies size + double links } & \multicolumn{2}{|c|}{ verifies bag/set + double links } \\
\hline append & 0.05 & 0.16 & 28.18 & 0.83 & 23.73 & 0.93 \\
\hline flatten (from tree) & 0.08 & 0.30 & 158.3 & 6.65 & 55.78 & 2.03 \\
\hline Sorted List & & \multicolumn{3}{|c|}{ verifies size $+\min +\max +$ sortedness } & \multicolumn{2}{|c|}{ verifies bag/set + sortedness } \\
\hline delete & 0.02 & 0.13 & 34.09 & 26.68 & 51.39 & 0.60 \\
\hline insertion_sort & 0.07 & 0.27 & 41.17 & 18.22 & 27.34 & 0.73 \\
\hline selection_sort & 0.10 & 0.41 & 79.08 & 20.62 & 221.7 & 1.10 \\
\hline bubble_sort & 0.16 & 0.64 & 358.7 & 9.36 & 221.2 & 2.84 \\
\hline merge_sort & 0.11 & 0.61 & 342.9 & 105.1 & 150.1 & 21.75 \\
\hline quick_sort & 0.19 & 0.59 & 642.0 & out of memory & failed & 3.40 \\
\hline Binary Search Tree & & \multicolumn{3}{|c|}{ verifies $\min +\max +$ sortedness } & \multicolumn{2}{|c|}{ verifies bag/set + sortedness } \\
\hline insert & 0.03 & 0.20 & failed & 11.92 & 99.57 & 0.95 \\
\hline delete & 0.06 & 0.38 & 97.5 & 6.86 & 943.5 & 3.03 \\
\hline Priority Queue Heap & & \multicolumn{3}{|c|}{ verifies size + height + max-heap } & \multicolumn{2}{|c|}{ verifies bag/set + size + max-heap } \\
\hline insert & 0.15 & 0.45 & 520.8 & 41.55 & 416.2 & 6.45 \\
\hline delete_max & 0.55 & 7.17 & failed & 290.7 & failed & 626.1 \\
\hline AVL Tree & & \multicolumn{3}{|c|}{ verifies size + height + height-balanced } & \multicolumn{2}{|c|}{ verifies bag/set + height + height-balanced } \\
\hline insert & 1.04 & 5.06 & failed & 36.02 & 1973 & 7.38 \\
\hline Red-Black Tree & & \multicolumn{3}{|c|}{ verifies size + black-height + height-balanced } & \multicolumn{2}{|c|}{ verifies bag/set + black-height + height-balanced } \\
\hline insert & 0.44 & 1.53 & 2992 & 352.4 & failed & 392.8 \\
\hline
\end{tabular}

Figure 6. Verification Times (in seconds) for Data Structures with Arithmetic and Bag/Set Constraints

property, their algorithm is able to handle AVL-like tree structures. Even then, the author acknowledged the lack of a general specification mechanism for handling arbitrary shape/size properties.

Size Properties. In another direction of research, size properties have been most explored for declarative languages $[10,28,6]$ as the immutability property makes their data structures easier to analyse statically. Size analysis was later extended to object-based programs [7] but was restricted to tracking either size-immutable objects that can be aliased and size-mutable objects that are unaliased, with no support for complex shapes. The Applied Type System (ATS) [5] was proposed for combining programs with proofs. In ATS, dependent types for capturing program invariants are extremely expressive and can capture many program properties with the help of accompanying proofs. Using linear logic, ATS may also handle mutable data structures with sharing. However, users must supply all expected properties, and precisely state where they are to be applied, with ATS playing the role of a proof-checker. Comparatively, we use a more limited class of constraint for shape and size analysis but supports automated modular verification.
Set/Bag Properties. Set-based analysis has been proposed to verify data structure consistency properties in [14], where a decision procedure is given for a first order theory that combines set and Presburger arithmetic. This result may be used to build a specialised mixed constraint solver but currently has high algorithmic complexity. In [15], Lahiri and Qadeer reported an intra-procedural reachability analysis for well-founded linked lists using first-order axiomatization. Reachability analysis is related to set/bag property that we capture but implemented by transitive closure at the predicate level.

Unfold/Fold Mechanism. Unfold/fold techniques were originally used for program transformation [4] on purely functional programs. A similar technique called unroll/roll was later used in alias types [27] to manually witness the isomorphism between a recursive type and its unfolding. Here, each unroll/roll step must be manually specified by programmer, in contrast to our approach which applies these steps automatically during entailment checking. In [1], an automated procedure that uses unroll/roll was given but it was hardwired to work for only lseg and tree predicates. Furthermore, it performs rolling by unfolding a pred- 
icate in the consequent which would miss bindings on free variables. Our unfold/fold mechanism is general, automatic and terminates for heap entailment checking.

\section{Conclusion}

We have presented a new approach to verifying pointerbased programs that can precisely track shape, size and bag properties. Our approach is built on well-founded shape relations and well-formed separation constraints from which we have designed a sound procedure for heap entailment. Our automated deduction mechanism is based on the unfold/fold reasoning of user-definable predicates that has been proven to be sound and terminating.

\section{Acknowledgement}

This work is supported by the Singapore-MIT Alliance and NUS research grant R-252-000-213-112.

\section{References}

[1] J. Berdine, C. Calcagno, and P. W. O'Hearn. Symbolic Execution with Separation Logic. In APLAS. Springer-Verlag, November 2005.

[2] J. Berdine, C. Calcagno, and P. W. O'Hearn. Smallfoot: Modular automatic assertion checking with separation logic. In FMCO, Springer LNCS 4111, 2006.

[3] J. Bingham and Z. Rakamaric. A Logic and Decision Procedure for Predicate Abstraction of Heap-Manipulating Programs. In VMCAI, Springer LNCS 3855, pages 207-221, Charleston, U.S.A, January 2006.

[4] R.M. Burstall and J. Darlington. A transformation system for developing recursive programs. Journal of ACM, 24(1):4467, January 1977.

[5] C. Chen and H. Xi. Combining Programming with Theorem Proving. In ACM SIGPLAN ICFP, Tallinn, Estonia, September 2005 .

[6] W.N. Chin and S.C. Khoo. Calculating sized types. In ACM SIGPLAN PEPM, pages 62-72, Boston, United States, January 2000.

[7] W.N. Chin, S.C. Khoo, S.C. Qin, C. Popeea, and H.H. Nguyen. Verifying Safety Policies with Size Properties and Alias Controls. In ACM SIGSOFT ICSE, St. Louis, Missouri, May 2005.

[8] D. Distefano, P. W. O'Hearn, and H. Yang. A Local Shape Analysis based on Separation Logic. In TACAS. SpringerVerlag, March 2006.

[9] A. Gotsman, J. Berdine, and B. Cook. Interprocedural Shape Analysis with Separated Heap Abstractions. In $S A S$, Springer LNCS, Seoul, Korea, August 2006.
[10] J. Hughes, L. Pareto, and A. Sabry. Proving the correctness of reactive systems using sized types. In ACM POPL, pages 410-423. ACM Press, January 1996.

[11] S. Isthiaq and P.W. O'Hearn. BI as an assertion language for mutable data structures. In ACM POPL, London, January 2001.

[12] L. Jia and D. Walker. ILC: A foundation for automated reasoning about pointer programs. In 15th ESOP, March 2006.

[13] Nils Klarlund and Anders Mller. Mona version 1.4 - user manual.

[14] V. Kuncak, H. H. Nguyen, and M. Rinard. An algorithm for deciding bapa: Boolean algebra with presburger arithmetic. In 20th International Conference on Automated Deduction (CADE-20), Tallinn, Estonia, Jul 2005.

[15] S. Lahiri and S. Qadeer. Verifying Properties of WellFounded Linked Lists. In ACM POPL, South Carolina, January 2006.

[16] O. Lee, H. Yang, and K. Yi. Automatic verification of pointer programs using grammar-based shape analysis. In ESOP. Springer Verlag, April 2005.

[17] M.J.Parkinson and G.M.Bierman. Separation logic and abstraction. In ACM POPL, pages 247-258, 2005.

[18] A. Moeller and M. I. Schwartzbach. The Pointer Assertion Logic Engine. In ACM PLDI, June 2001.

[19] H.H. Nguyen, C. David, S.C. Qin, and W.N. Chin. Automated Verification of Shape, Size and Bag Properties via Separation Logic. Technical report, SoC, Natl Univ. of Singapore, July 2006. avail. at http://www.comp.nus.edu.sg/ $\sim$ chinwn/papers/verify-report.pdf.

[20] H.H. Nguyen, C. David, S.C. Qin, and W.N. Chin. Automated Verification of Shape And Size Properties via Separation Logic. In VMCAI, Nice, France, January 2007.

[21] Tobias Nipkow, Lawrence C. Paulson, and Markus Wenzel. Isabelle/HOL - A Proof Assistant for Higher-Order Logic, volume 2283 of LNCS. Springer, 2002.

[22] W. Pugh. The Omega Test: A fast practical integer programming algorithm for dependence analysis. Communications of the ACM, 8:102-114, 1992.

[23] J. Reynolds. Separation Logic: A Logic for Shared Mutable Data Structures. In IEEE LICS, Copenhagen, Denmark, July 2002.

[24] R. Rugina. Quantitative Shape Analysis. In SAS, Springer LNCS, Verona, Italy, August 2004.

[25] S. Sagiv, T. Reps, and R. Wilhelm. Parametric shape analysis via 3-valued logic. ACM TOPLAS, 24(3), May 2002.

[26] É-J. Sims. Extending separation logic with fixpoints and postponed substitution. Theoretical Computer Science, 351(2):258-275, 2006.

[27] D. Walker and G. Morrisett. Alias Types for Recursive Data Structures. In TIC, Springer LNCS 2071, pages 177-206, 2000.

[28] H. Xi. Dependent Types in Practical Programming. PhD thesis, Carnegie Mellon University, 1998. 